\title{
Age-Related ultrasound changes in muscle quantity and quality in women
}

\section{$\operatorname{AUTHOR}(S):$}

Fukumoto, Yoshihiro; Ikezoe, Tome; Yamada, Yosuke; Tsukagoshi, Rui; Nakamura, Masatoshi; Takagi, Yui; Kimura, Misaka; Ichihashi, Noriaki

\section{CITATION:}

Fukumoto, Yoshihiro ...[et al]. Age-Related ultrasound changes in muscle quantity and quality in women. Ultrasound in Medicine and Biology 2015, 41(11): 3013-3017

\section{ISSUE DATE:}

2015-11

URL:

http://hdl.handle.net/2433/231219

\section{RIGHT:}

(c) 2015. This manuscript version is made available under the CC-BY-NC-ND 4.0 license

http://creativecommons.org/licenses/by-nc-nd/4.0/ The full-text file will be made open to the public on 01 November 2016 in accordance with publisher's 'Terms and Conditions for Self-Archiving'. This is not the published version. Please cite only the published version.この論文は出版社版でありません。引用の際には出版社版をご確認ざ利用ください。 


\section{Age-Related Ultrasound Changes in Muscle Quantity and Quality in Women}

Yoshihiro Fukumoto ${ }^{1,2}$, Tome Ikezoe ${ }^{2}$, Yosuke Yamada ${ }^{3}$, Rui Tsukagoshi ${ }^{4}$, Masatoshi Nakamura ${ }^{2,5}$, Yui Takagi ${ }^{6}$,

Misaka Kimura ${ }^{7}$, and Noriaki Ichihashi ${ }^{2}$

${ }^{1}$ Faculty of Rehabilitation, Kobe Gakuin University, Kobe, Japan

${ }^{2}$ Human Health Sciences, Graduate School of Medicine, Kyoto University, Kyoto, Japan

${ }^{3}$ Department of Nutritional Science, National Institute of Health and Nutrition, Tokyo, Japan

${ }^{4}$ School of Rehabilitation, Hyogo University of Human Science, Kobe, Japan

${ }^{5}$ Faculty of Health and Sports Science, Doshisha University, Kyotanabe, Kyoto, Japan

${ }^{6}$ Department of Rehabilitation, Nagoya University Hospital, Nagoya, Japan

${ }^{7}$ Faculty of Bioenvironmental Science, Kyoto Gakuen University, Kameoka, Kyoto, Japan

Corresponding author : Yoshihiro Fukumoto, PhD,

Faculty of Rehabilitation, Kobe Gakuin University

518 Arise, Ikawadani-cho, Nishi-ku, Kobe 651-2180, Japan

Phone: +81-78-974-2461

Fax: +81-78-974-2461

E-mail: fukumoto@reha.kobegakuin.ac.jp 


\section{Abstract}

2 This study investigated the age-related changes in muscle quantity and quality in the trunk and limbs in women. A total of

3128 females were classified into 4 age groups: young, middle-aged, young-old and old-old. Muscle thickness (MT) and

4 echo intensity (EI) were measured for the biceps brachii, quadriceps femoris, rectus abdominis, external oblique, internal

5 oblique and transversus abdominis, using B-mode ultrasonography. The EIs of the biceps brachii, quadriceps femoris and transversus abdominis were significantly higher in the middle-aged group than in the young group; however, there were no significant differences in MT. Compared with the young group, all other groups had significant changes in both the MT and EI of the rectus abdominis, external oblique and internal oblique muscles. Thus, qualitative changes in muscle may

9 occur earlier than quantitative changes, and loss of muscle mass in the superficial abdominal muscles may occur earlier

10 than those in the other muscles.

11 Keywords: muscle quantity, muscle quality, ultrasonography, trunk muscles 


\section{Introduction} considered as a primary reason of loss of independence and falls (Wolfson et al. 1995). Previous studies have typically assessed age-related loss of muscle mass through muscle volume or cross-sectional area using magnetic resonance imaging

5 (Janssen et al. 2000), or computed tomography (Overend et al. 1992), and muscle thickness (MT) using ultrasound

6 (Candow and Chilibeck 2005; Arts et al. 2007). However, actual loss of contractile muscle tissue with aging is greater than the decrease in muscle size assessed using these standard imaging methods because they are affected by changes in muscle quality or composition including fat, connective tissue and extracellular water content (Yamada et al. 2010; Fukumoto et

9 al. 2012b).

10 Recently, it has become possible to assess muscle quality in vivo by non-invasive ultrasound imaging in which an

11 enhanced echo intensity (EI) represents augmented fat and connective tissue within muscle (Pillen et al. 2009). Previous studies by others and us have assessed MT and EI as indices of muscle quantity and quality in the limbs; these revealed

13 that decreases in MT and increases in EI occurred with aging (Arts et al. 2010; Fukumoto et al. 2012b). Thus, quantitative

14 and qualitative changes are known to occur in the limb muscles with age. However, no study has investigated the correlation

15 between the trunk and limb muscle changes with aging. Therefore, this study aimed to investigate the age-related changes

16 in muscle quantity and quality in the upper and lower limbs and trunk muscles in women.

19 A total of 128 women participated in this study after providing written informed consent. Of these, 28 were young (age 

19-30 years) and 100 were community-dwelling, middle-aged and elderly women living independently (age, 53-92 years).

2 The middle-aged and elderly participants were divided into 3 categories: middle-aged ( $<65$ years), young-old ( $65-74$ years)

3 and old-old ( $\geq 75$ years). The eligibility criteria were as follows: able to walk without assistive devices and climb stairs, no dementia, no history of trauma, surgery, neuromuscular disorders, metabolic disorders, or acute and chronic diseases that impair the muscle quantity, quality and strength, or physical function. The history of parturition (yes or no) was recorded using a questionnaire. The ethics committee of Kyoto University Graduate School and the Faculty of Medicine approved the study protocol.

9 Chalfont, Buckinghamshire, UK) with an $8 \mathrm{MHz}$ linear-array probe. Equipment settings were consistently set to the following: gain (58 dB), dynamic range $(69 \mathrm{~Hz})$ and time gain compensation in the neutral position. Transverse ultrasound

11 images of 6 muscles were obtained on the right side as previously described (Arts et al. 2010; Fukumoto et al. 2012a) from the biceps brachii (including the underlying brachialis), quadriceps femoris, rectus abdominis, external oblique, internal oblique and transversus abdominis muscles. The measurement site for each muscle are shown in Figure 1. During measurements, the participants were asked to lay in the supine position with their arms and legs extended and their muscles completely relaxed. All examinations were performed once for each muscle with great care by the same experienced investigator. Representative ultrasound images are shown in Figure 2. further analysis. MT was assessed as an index of muscle quantity using an electric caliper on the ultrasound images. EI was 
1 in Adobe Photoshop Elements (Adobe Systems Inc., San Jose, California, USA). The method and its reliability have been

2 described in more detail in other studies (Arts et al. 2010; Fukumoto et al. 2012a; Ota et al. 2012).

3 Results are presented as the mean \pm standard deviation. Group differences were analyzed using one-way analysis of variance followed by Tukey’s post hoc test. All analyses were performed using IBM SPSS 20.0 for Windows (IBM Corp.,

5 Armonk, New York, USA). P values of $<0.05$ were considered statistically significant.

\section{Results}

Table 1 summarises the physical characteristics of the participants. Compared with the young group, BMI was

9 significantly higher in the middle-age and the young-old groups $(\mathrm{p}<0.05)$, but not in the old-old group. Table 2 summarises

the results of the ultrasound measurements. There were significant main effects in MT and EI for all muscles measured (p

$11<0.05)$.

MT of the biceps brachii showed a significant difference only between the middle-aged and old-old groups $(\mathrm{p}<$

13 0.05). MT of the quadriceps femoris was significantly thinner in the young-old and old-old groups than in the young group,

14 and that was significantly thinner in the old-old group than the middle-aged group $(\mathrm{p}<0.01)$. Compared with the young

15 group, MT of the rectus abdominis, external oblique and internal oblique were significantly thinner in the other groups (p

$16<0.01)$. MT in the transversus abdominis showed a significant difference only between the young and the young-old groups

$17 \quad(\mathrm{p}<0.01)$. 
1 oblique in the old-old group than in the middle-aged group ( $\mathrm{p}<0.05)$. No significant differences were observed in EI of

2 the other muscles among the middle-aged, young-old and old-old groups.

Discussion

Generally, age-related muscle atrophy is greater in the lower than the upper limbs (Janssen et al. 2000; Arts et al. 2007). Our results also demonstrated that the MT of the quadriceps femoris in young-old and old-old adults decreased when compared with those of that in young adults, while the biceps brachii showed smaller age-related atrophy. In contrast to our data, Candow and Chilibeck (2005) have reported that the MT of the elbow flexors and knee extensors in older men (59-76 years) were significantly thinner than those of younger men (18-31 years). A possible explanation for this discrepancy is that age-related muscle atrophy may be more marked in men than women (Janssen et al. 2000). et al. 2010; Fukumoto et al. 2012b). Our study further examined this age-related change dividing the subjects into four age

13 groups, and clarified that the change in EI starts from the middle age, and that this change in these muscles occur prior to

14 the change in MT, when no significant decrease in MT is yet seen. Thus, age-related changes in muscle quality may occur

15 earlier than losses of muscle quantity during the life span. These results indicate that adipose and connective tissue within

16 this muscle had increased, resulting in a substantial decrease in contractile tissue even in middle age. Physiological evidence

17 suggests that increased fat and fibrous tissue within the muscle is associated with poor muscle strength (Fukumoto et al.

onward. 
Previous studies have reported age-related losses of the abdominal muscles mass (Kanehisa et al. 2004; Ota et al.

2 2012). Notably, Ota et al. (2012) reported that MT of the superficial abdominal muscles, such as the rectus abdominis,

3 external oblique and internal oblique, was thinner among middle-aged women than younger women, whereas there was no corresponding decrease in the MT of the deep abdominal muscles, such as the transversus abdominis. Although our study is consistent with these results, we additionally revealed that the MT of the superficial abdominal muscles decreased from middle age without finding differences in the MT of the biceps brachii or quadriceps femoris muscles between young and middle-aged women. These results suggest that age-related atrophy of the superficial abdominal muscles may occur earlier than that of the limb muscles in middle age. Furthermore, the EI was higher in all the trunk muscles of the middle-aged group when compared with the young group. Thus, we revealed that both quantitative and qualitative changes occurred in the superficial abdominal muscles of middle-aged women. Notably, although age-related atrophy was reported to be less

11 in the transversus abdominis (Ota et al. 2012), it was clarified that the actual contractile tissue in this muscle is decreased by augmented fat and connective tissue. Impairment in the trunk muscles can result in a loss of stability to the lumbo-pelvic region (Urquhart et al. 2005). These results suggest that intervention that improves the quantity and quality of trunk muscles may be needed from middle age. However, given that the differences in MT and EI of the trunk muscles were generally insignificant among the middle-aged, young-old and old-old groups, it is possible that the quantity and quality of these muscles is maintained beyond middle age. These findings may be affected by the history of parturition. No women in the young group had experienced parturition, whereas $77 \%$ of the total participants in the middle-aged and elderly groups had experienced it. Further study is required to clarify the effects of parturition. 
1 ultrasound imaging is a low-cost, easy-to-operate and safe method, it is suitable in the clinical setting to evaluate

2 quantitative and qualitative changes in various muscles with aging. Changes in the muscle quantity and quality have also

3 been reported in patients with lower-limb osteoarthritis (Fukumoto et al. 2012a), metabolic disorders (Koster and Schaap

4 2015), or following stroke (Lee et al. 2015). Further studies using ultrasound to measure MT and EI and clarify muscle

$5 \quad$ characteristics of various diseases are required in future.

The present study has several limitations. One limitation was that the sample size in our study was small. Notably, we did not included women aged 30-40 years due to difficulty in recruitment, during which age-related increases in the EI might occur in the superficial abdominal muscles. However, in future, we aim to include a large number of women of all

9 ages over 18, including 30-40. Another limitation of this study was its cross-sectional design and that we included only

11 measured only the right side. This was feasible because we excluded subjects whose muscle characteristics were expected to be asymmetrical. However, it has been demonstrated that there was asymmetry between the left and right sides in the

13 muscle thickness of biceps brachii (Arts et al. 2010). Therefore, the results of muscle quantity and quality, particularly for

14 biceps brachii, may have been affected depending on whether or not measurements were taken from the side of the

15 dominant hand. In conclusion, age-related changes in muscle quality may occur at an earlier age than losses of muscle quantity. In

17 addition, loss of muscle mass in the superficial abdominal muscles, such as the rectus abdominis, external oblique and

18 internal oblique, may occur from an earlier age than the loss of muscle mass in the limb and the deep abdominal muscles. 


\section{References}

Arts IM, Pillen S, Overeem S, Schelhaas HJ, Zwarts MJ. Rise and fall of skeletal muscle size over the entire life

span. J Am Geriatr Soc 2007;55:1150-2.

Arts IM, Pillen S, Schelhaas HJ, Overeem S, Zwarts MJ. Normal values for quantitative muscle ultrasonography in adults. Muscle Nerve 2010;41:32-41.

young and older men. J Gerontol A Biol Sci Med Sci 2005;60:148-56. consensus on definition and diagnosis: Report of the European Working Group on Sarcopenia in Older People. Age Ageing

Med Biol 2012a;38:1540-5. 
subcutaneous fat thickness. Eur J Appl Physiol 2004;91:534-7.

Koster A, Schaap LA. The effect of type 2 diabetes on body composition of older adults. Clin Geriatr Med

Lee SS, Spear S, Rymer WZ. Quantifying changes in material properties of stroke-impaired muscle. Clin Biomech

Ota M, Ikezoe T, Kaneoka K, Ichihashi N. Age-related changes in the thickness of the deep and superficial

abdominal muscles in women. Arch Gerontol Geriatr 2012;55:e26-30.

Overend TJ, Cunningham DA, Kramer JF, Lefcoe MS, Paterson DH. Knee extensor and knee flexor strength: cross-

sectional area ratios in young and elderly men. J Gerontol 1992;47:M204-10.

Pillen S, Tak RO, Zwarts MJ, Lammens MM, Verrijp KN, Arts IM, van der Laak JA, Hoogerbrugge PM, van

Engelen BG, Verrips A. Skeletal muscle ultrasound: correlation between fibrous tissue and echo intensity. Ultrasound Med

Biol 2009;35:443-6.

Urquhart DM, Hodges PW, Allen TJ, Story IH. Abdominal muscle recruitment during a range of voluntary exercises.

Man Ther 2005;10:144-53.

Wolfson L, Judge J, Whipple R, King M. Strength is a major factor in balance, gait, and the occurrence of falls. J

Gerontol A Biol Sci Med Sci 1995;50 Spec No:64-7. 
Table 1. Physical characteristics of the participants $(n=128)$

\begin{tabular}{|c|c|c|c|c|}
\hline & Young $(\mathrm{n}=28)$ & Middle-aged $(\mathrm{n}=25)$ & Young-old $(\mathrm{n}=49)$ & Old-old $(n=26)$ \\
\hline Age (years) & $21.5 \pm 2.6(19 ; 30)$ & $60.5 \pm 3.1(53 ; 64)$ & $69.8 \pm 2.8(65 ; 74)$ & $79.3 \pm 4.3(75 ; 92)$ \\
\hline Height (cm) & $161.2 \pm 4.7(153.0 ; 172.0)$ & $154.5 \pm 5.8(140.1 ; 162.8) * *$ & $151.2 \pm 5.1(137.1 ; 160.9) * *$ & $150.0 \pm 6.1(140.9 ; 164.9) * * \dagger$ \\
\hline Weight (kg) & $54.6 \pm 5.6(45.0 ; 66.2)$ & $54.6 \pm 7.9(44.1 ; 70.8)$ & $52.0 \pm 7.2(37.0 ; 66.8)$ & $48.6 \pm 5.5(39.0 ; 64.0) * *$ \\
\hline BMI $\left(\mathrm{kg} / \mathrm{m}^{2}\right)$ & $21.0 \pm 1.8(16.7 ; 24.1)$ & $22.9 \pm 2.9(18.1 ; 29.9) *$ & $22.7 \pm 2.6(17.0 ; 28.8) *$ & $21.6 \pm 1.7$ (18.9; 26.3) \\
\hline Parturition (yes/no), n (\%) & $0(0) / 28(100)$ & $21(84) / 4(16)$ & $40(81.6) / 9(18.4)$ & $16(61.5) / 10(38.5)$ \\
\hline
\end{tabular}

Data presented as mean \pm standard deviations (minimum; maximum). BMI; Body mass index

Significant difference compared with young $\left({ }^{*} \mathrm{p}<0.05\right.$, **p $\left.<0.01\right)$

Significant difference compared with middle-aged $(\dagger p<0.05, \neq p<0.01)$ 
Table 2. Results of ultrasound measurements $(n=128)$

\begin{tabular}{|c|c|c|c|c|}
\hline & Young $(\mathrm{n}=28)$ & Middle-aged (n = 25) & Young-old $(n=49)$ & Old-old (n = 26) \\
\hline \multicolumn{5}{|l|}{$\mathrm{MT}(\mathrm{cm})$} \\
\hline Biceps brachii & $2.36 \pm 0.27$ & $2.46 \pm 0.34$ & $2.28 \pm 0.31$ & $2.21 \pm 0.29 \dagger$ \\
\hline Quadriceps femoris & $4.55 \pm 0.53$ & $4.12 \pm 0.69$ & $3.81 \pm 0.56 * *$ & $3.45 \pm 0.71 * * \neq$ \\
\hline Rectus abdominis & $1.09 \pm 0.18$ & $0.81 \pm 0.18 * *$ & $0.76 \pm 0.16 * *$ & $0.70 \pm 0.17 * *$ \\
\hline External oblique & $0.79 \pm 0.14$ & $0.67 \pm 0.19 *$ & $0.58 \pm 0.14 * *$ & $0.52 \pm 0.14 * * \neq$ \\
\hline Internal oblique & $1.13 \pm 0.27$ & $0.78 \pm 0.21 * *$ & $0.79 \pm 0.25 * *$ & $0.79 \pm 0.18 * *$ \\
\hline Transversus abdominis & $0.44 \pm 0.14$ & $0.37 \pm 0.10$ & $0.34 \pm 0.11 * *$ & $0.39 \pm 0.10$ \\
\hline \multicolumn{5}{|l|}{ EI (0-255) } \\
\hline Biceps brachii & $86.6 \pm 9.7$ & $96.7 \pm 14.6$ * & $106.3 \pm 13.5 * * \dagger$ & $108.7 \pm 9.3 * * \ddagger$ \\
\hline Quadriceps femoris & $78.4 \pm 5.2$ & $99.9 \pm 9.4 * *$ & $101.8 \pm 9.3 * *$ & $109.7 \pm 9.4 * * \neq \S \S$ \\
\hline Rectus abdominis & $62.0 \pm 13.0$ & $117.7 \pm 17.7 * *$ & $127.4 \pm 17.6 * *$ & $124.8 \pm 17.5 * *$ \\
\hline External oblique & $79.6 \pm 9.3$ & $115.7 \pm 11.9 * *$ & $120.7 \pm 12.6$ ** & $125.7 \pm 10.5 * * \dagger$ \\
\hline Internal oblique & $59.6 \pm 9.8$ & $96.5 \pm 14.5 * *$ & $101.9 \pm 14.3^{* *}$ & $104.3 \pm 14.2 * *$ \\
\hline Transversus abdominis & $46.0 \pm 13.1$ & $80.3 \pm 13.3 * *$ & $86.4 \pm 17.0 * *$ & $87.7 \pm 15.3 * *$ \\
\hline
\end{tabular}

Data presented as mean \pm standard deviations. MT; Muscle thickness, EI; Echo intensity

Significant difference compared with young $\left({ }^{*} \mathrm{p}<0.05,{ }^{* *} \mathrm{p}<0.01\right)$

Significant difference compared with middle-aged $(\dagger \mathrm{p}<0.05$, $\neq \mathrm{p}<0.01)$

Significant difference compared with young-old $(\S \S \mathrm{p}<0.01)$ 


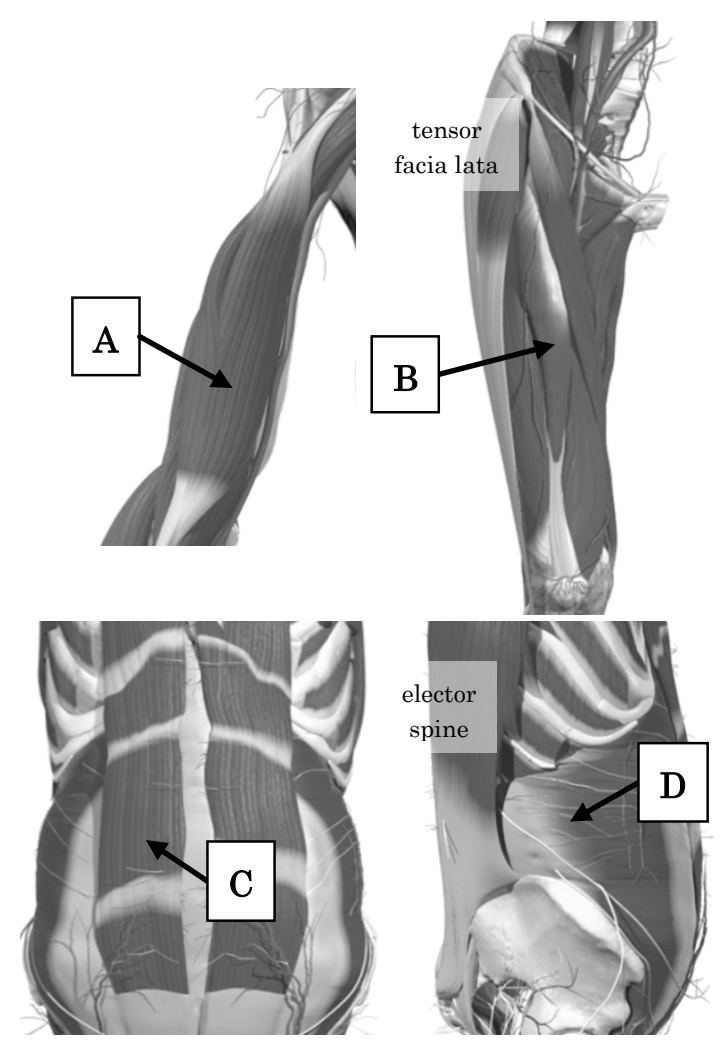

Fig 1. Ultrasound sites for each muscle. A: Biceps brachii, Two-thirds between the acromion and the antecubital crease. B: Quadriceps femoris, Midway between the anterior superior iliac spine and the proximal end of the patella. C: Rectus abdomimis, $3 \mathrm{~cm}$ lateral to the umbilicus. D: External oblique, internal oblique and transversus abdominis, $2.5 \mathrm{~cm}$ anterior to the mid-axillary line, at the mid-point between the inferior rib and the iliac crest. (These figures were cited from Primal Pictures on OvidSP and Fukumoto et al. 2012a.) 


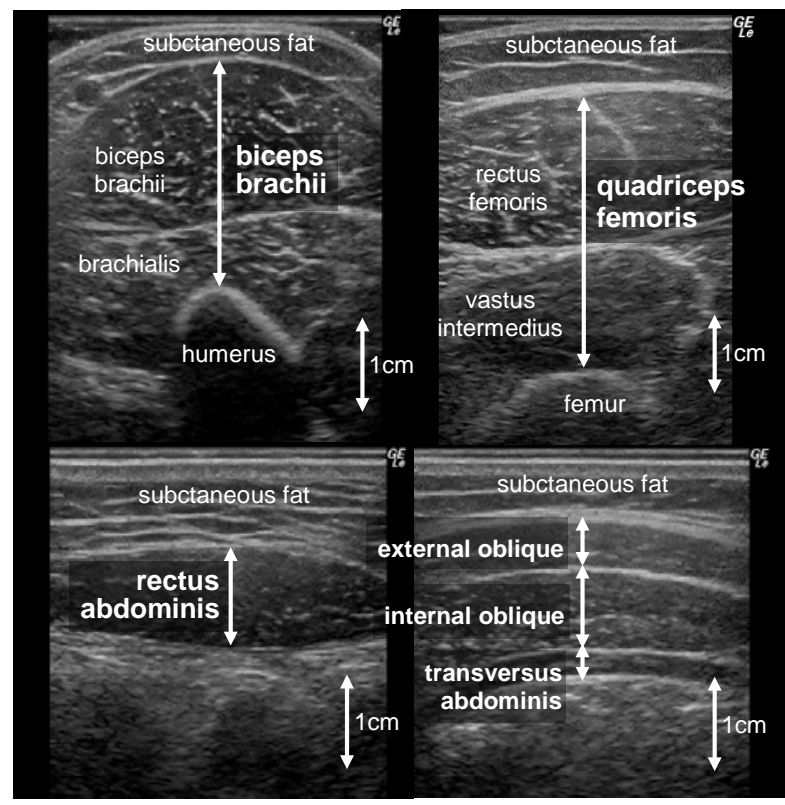

Fig 2. Representative ultrasound images. 